Theorem 7. The set a is a modular ideal in $L$ containing the set $M_{L}^{\prime}$.

Proof. It is obvious that $\mathfrak{a}$ is an ideal; that it is modular follows from the corollary to Theorem 5, equations (7) and Lemma 2. Now if $(b, c) M^{\prime}$, then by Lemma $2 b \otimes c \notin L$, whence $b c=(b \otimes c) \otimes \infty$, and $b c \in \mathfrak{a}$. Hence $M_{L}^{\prime} \subset \mathfrak{a}$.

It should be observed that both possibilities $M_{L}^{\prime}=\mathfrak{a}$ and $M_{L}^{\prime} \neq \mathfrak{a}$ can occur. In our special example where $\Lambda$ is a projective geometry and $\Lambda-L$ consists of all subelements $x$ of a hyperplane $h$ with $x$ not $\leqq \infty, M_{L}^{\prime}=\mathfrak{a}$ if there exists $k \notin L$ with $\infty<k<h$. On the other hand, if no element exists (in $\Lambda$ ) between $\infty$ and $h$, then $M_{L}^{\prime} \neq \mathfrak{a}$, for then $\infty \notin M_{L}^{\prime}$. This example shows also that $M_{L}^{\prime}$ need not be an ideal.

IllinoIs Institute of TEChNOLOGY

\title{
SUFFICIENT CONDITIONS THAT POLYNOMIALS IN SEVERAL VARIABLES BE POSITIVE
}

\author{
IRWIN E. PERLIN
}

1. Introduction. Let $T(x)$ denote a polynomial in a single real variable $x$ with real coefficients. T. Popoviciu ${ }^{1}$ established sufficient conditions that $T(x)$ be positive for all real $x$. In this paper we shall consider polynomials in several real variables with real coefficients, and establish sufficient conditions that the polynomials be positive for real values of the variables.

2. Polynomials in two real variables. In this section we shall develop sufficient conditions that a polynomial in two real variables with real coefficients be positive for all real values of the variables. Let us consider then,

$$
T(x, y)=\sum_{i=0}^{2 m} \sum_{j=0}^{2 n} b_{i j} c_{i j} x^{i} y^{j},
$$

where the $b_{i j}$ are positive constants, and the $c_{i j}$ are real numbers. Introducing parameters, we write $T(x, y)$ in the following form

Presented to the Society, April 13, 1940; received by the editor June 26, 1941.

$1 \mathrm{~T}$. Popoviciu, Sur une condition suffisante pour qu'un polynôme soit positif, Mathematica, vol. 11 (1935), pp. 247-256. 


$$
\begin{aligned}
& T(x, y)=\sum_{i=0}^{m-1} \sum_{j=0}^{n-1}\left\{\alpha _ { i j } c _ { 2 i } { } _ { 2 j } x ^ { 2 i } y ^ { 2 j } \left[\left(1+\beta_{i j} \frac{c_{2 i+12 j}}{c_{2 i 2 j}} x\right)^{2}\right.\right. \\
& \left.+\left(1+\gamma_{i j} \frac{c_{2 i 2 j+1}}{c_{2 i 2 j}} y\right)^{2}\right] \\
& +\zeta_{i j} \frac{\left(1 / \lambda^{2}\right) c_{2 i 2 j} c_{2 i+22 j}-c_{2 i+12 j}^{2}}{c_{2 i 2 j}} x^{2 i+2} y^{2 j} \\
& \left.+\eta_{i j} \frac{\left(1 / \lambda^{2}\right) c_{2 i 2 j} c_{2 i 2 j+2}-\stackrel{2}{c_{2 i 2 j+1}}}{c_{2 i 2 j}} x^{2 i} y^{2 j+2}\right\} \\
& +\sum_{j=0}^{n-1}\left\{2 \alpha_{m j} c_{2 m 2 j} x^{2 m} y^{2 j}\left(1+\gamma_{m j} \frac{c_{2 m 2 j+1}}{2 c_{2 m 2 j}} y\right)^{2}\right. \\
& \left.+\eta_{m j} \frac{\left(1 / \lambda^{2}\right) c_{2 m 2 j} c_{2 m 2 j+2}-(1 / 2) c_{2 m 2 j+1}^{2}}{c_{2 m 2 j}} x^{2 m} y^{2 j+2}\right\} \\
& +\sum_{i=0}^{m-1}\left\{2 \alpha_{i n} c_{2 i 2 n} x^{2 i} y^{2 n}\left(1+\beta_{i n} \frac{c_{2 i+12 n}}{2 c_{2 i 2 n}} x\right)^{2}\right. \\
& \left.+\zeta_{i n} \frac{\left(1 / \lambda^{2}\right) c_{2 i 2 n} c_{2 i+22 n}-(1 / 2) c_{2 i+12 n}^{2}}{c_{2 i 2 n}} x^{2 i+2} y^{2 n}\right\} \\
& +\sum_{i=0}^{m-1} \sum_{j=0}^{n-1} b_{2 i+1} 2_{j+1} c_{2 i+1}{ }_{2 j+1} x^{2 i+1} y^{2 j+1}+2 \alpha_{m n} c_{2 m 2 n} x^{2 m} y^{2 n} .
\end{aligned}
$$

By comparing coefficients, we find

$$
\begin{aligned}
& 2 \alpha_{00}=b_{00}, \\
& 2 \alpha_{i j} \beta_{i j}=b_{2 i+12 j}, \quad \zeta_{i j}=\beta_{i j}^{2} \alpha_{i j}, \\
& i=0,1, \cdots, m-1 ; j=0,1, \cdots, n \text {, } \\
& 2 \alpha_{i j} \gamma_{i j}=b_{2 i 2 i+1}, \quad \eta_{i j}={ }^{2} \gamma_{i j} \alpha_{i j} \text {, } \\
& i=0,1, \cdots, m ; j=0,1, \cdots, n-1 \text {, } \\
& \frac{1}{\lambda^{2}} \zeta_{i-10}+2 \alpha_{i 0}=b_{2 i 0}, \quad i=1,2, \cdots, m \text {, } \\
& \frac{1}{\lambda^{2}} \eta_{0 j-1}+2 \alpha_{0 j}=b_{02 j}, \quad i=1,2, \cdots, n \\
& \frac{1}{\lambda^{2}} \zeta_{i-1 j}+\frac{1}{\lambda^{2}} \eta_{i j-1}+2 \alpha_{i j}=b_{2 i 2 j} \\
& i=1,2, \cdots, m ; j=1,2, \cdots, n \text {. }
\end{aligned}
$$


We define $\zeta_{-1 j}=\eta_{i-1}=0(i=0,1, \cdots, m ; j=0,1, \cdots, n)$. The last three equations can be written

$$
\begin{aligned}
\frac{1}{\lambda^{2}} \zeta_{i-1 i}+\frac{1}{\lambda^{2}} \eta_{i j-1} & +2 \alpha_{i j}=b_{2 i 2 j}, \\
i & =0,1, \cdots, m ; j=0,1, \cdots, n .
\end{aligned}
$$

The parameters $\alpha_{i j}$ are determined by the recursion relations

$$
\alpha_{i j}=\frac{4 \lambda^{2} b_{2 i}{ }_{2 j} \alpha_{i-1 j} \alpha_{i j-1}-b_{2 i-1{ }_{2 j}}^{2} \alpha_{i j-1}-b_{2 i 2 j-1}^{2} \alpha_{i-1 j}}{8 \lambda^{2} \alpha_{i-1} \alpha_{i j-1}},
$$

where we have defined $b_{-1 j}=b_{i-1}=0$ and $\alpha_{i-1}=\alpha_{-1 j}=1(i=0,1, \cdots, m$; $j=0,1, \cdots, n)$. Let us write

$$
\alpha_{i j}=\frac{P_{i j}(\lambda)}{2 \lambda Q_{i j}(\lambda)},
$$

where $P_{i j}(\lambda)$ and $Q_{i j}(\lambda)$ are polynomials in $\lambda$. The polynomials $P_{i j}(\lambda)$ and $Q_{i j}(\lambda)$ satisfy the recursion relations

$$
\begin{aligned}
P_{00}(\lambda)= & b_{00} \lambda, \quad Q_{00}(\lambda)=1, \\
P_{i j}(\lambda)= & \lambda b_{2 i}{ }_{2 j} Q_{i j}(\lambda)-\frac{1}{2} b_{2 i-1}^{2} P_{2 j} P_{i-1}(\lambda) Q_{i-1}(\lambda) \\
& -\frac{1}{2} b_{2 i}^{2}{ }_{2 j-1} P_{i-1}(\lambda) Q_{i j-1}(\lambda), \\
Q_{i j}(\lambda)= & P_{i-1} j_{j}(\lambda) P_{i j-1}(\lambda),
\end{aligned}
$$

where we define $P_{-1 j}(\lambda)=P_{i-1}(\lambda)=Q_{-1 j}(\lambda)=Q_{i-1}(\lambda)=1(i=0,1, \cdots, m$; $j=0,1, \cdots, n)$. Let $\lambda_{i j}$ be the greatest positive zero of $P_{i j}(\lambda)$. Then we establish the following lemma.

LEMmA 1. $\bar{\lambda}_{i j}$ is greater than or equal to the greater of $\bar{\lambda}_{i-1 ;}$ and $\bar{\lambda}_{i j-1}$.

We proceed to prove this lemma by induction. Obviously $\bar{\lambda}_{10} \geqq \bar{\lambda}_{00}$ and $\bar{\lambda}_{01} \geqq \bar{\lambda}_{00}$. Since the degree of $P_{i-1}{ }_{j}(\lambda)$ is greater than that of $P_{i-1 j-1}(\lambda) P_{i-2}{ }_{j}(\lambda)$ and the degree of $P_{i j-1}(\lambda)$ is greater than that of $P_{i-1}{ }_{j-1}(\lambda) P_{i j-2}(\lambda)$, it follows that the degree of $P_{i j}(\lambda)$ is the same as that of $\lambda b_{2 i}{ }_{2 j} P_{i-1}{ }_{j}(\lambda) P_{i j-1}(\lambda)$. We see that the coefficient of the highest power of $\lambda$ in $P_{i j}(\lambda)$ is positive. Let $\tau$ equal the greater of $\bar{\lambda}_{i-1} j$ and $\bar{\lambda}_{i j-1}$. Then $P_{i j}(\tau) \leqq 0$. Hence, $\bar{\lambda}_{i j}$ is greater than or equal to the greater of $\bar{\lambda}_{i-1} j_{j}$ and $\bar{\lambda}_{i j-1}$.

THEOREM 1. Let $r$ be a real constant. If $r>\bar{\lambda}_{m n}$, and

$$
c_{00}>0,
$$




$$
\begin{aligned}
& \frac{1}{r^{2}} c_{2 i 2 j} c_{2 i+22 j}-c_{2 i+12 j}^{2}>0 \text {, } \\
& i=0,1, \cdots, m-1 ; j=0,1, \cdots, n-1, \\
& \frac{1}{r^{2}} c_{2 i 2 n} c_{2 i+2}-\frac{1}{2} c_{2 i+12 n}^{2}>0, \quad i=0,1, \cdots, m-1 \text {, } \\
& \frac{1}{r^{2}} c_{2 i 2 j} c_{2 i 2 j+2}-c_{2 i 2 j+1}^{2}>0 \\
& i=0,1, \cdots, m-1 ; j=0,1, \cdots, n-1, \\
& \frac{1}{r^{2}} c_{2 m 2 j} c_{2 m 2 j+2}-\frac{1}{2} c_{2 m 2 j+1}^{2}>0, \quad j=0,1, \cdots n,-1 \text {, } \\
& c_{2 i+12 j+1}=0, i=0,1, \cdots, \quad m-1 ; j=0,1 \cdots, n-1 .
\end{aligned}
$$

then $T(x, y)>0$ for all real values of $x$ and $y$.

By the preceding lemma $P_{i j}(r)>0(i=0,1, \cdots, m ; j=0,1, \cdots, n)$. Hence $\alpha_{i j}>0(i=0,1, \cdots, m ; j=0,1, \cdots, n), \zeta_{i j}>0(i=0,1, \cdots$, $m-1 ; j=0,1, \cdots, n)$, and $\eta_{i j}>0(i=0,1, \cdots, m ; j=0,1, \cdots, n-1)$. It follows that $T(x, y)>0$ for all real $x$ and $y$.

3. Applications. In this section we shall study the case where $b_{i j}=1$ $(i=0,1, \cdots, 2 m ; j=0,1, \cdots, 2 n)$, and deduce a sufficient condition that the polynomial

$$
L(x, y)=\sum_{i=0}^{2 m} \sum_{j=0}^{2 n} c_{i j} x^{i} y^{j}
$$

be positive for all real values of $x$ and $y$. For this case, the polynomials $P_{i j}(\lambda)$ satisfy the recursion relations

$$
\begin{aligned}
& P_{00}(\lambda)=\lambda, \\
& P_{i 0}(\lambda)=\lambda P_{i-10}(\lambda)-\frac{1}{2} P_{i-20}(\lambda), \\
& i=1,2, \cdots, m \text {, } \\
& P_{0 j}(\lambda)=\lambda P_{0 j-1}(\lambda)-\frac{1}{2} P_{0 j-2}(\lambda), \\
& j=1,2, \cdots, n \text {, } \\
& P_{i j}(\lambda)=\lambda P_{i-1 j}(\lambda) P_{i j-1}(\lambda)-\frac{1}{2} P_{\imath j-1}(\lambda) P_{i-1 j-1}(\lambda) P_{i-2}{ }_{j}(\lambda) \\
& -\frac{1}{2} P_{i-1 j}(\lambda) P_{i-1 j-1}(\lambda) P_{i j-2}(\lambda) \text {, } \\
& i=1,2, \cdots, m ; j=1,2, \cdots, n,
\end{aligned}
$$

wh ere we define $P_{-1 j}(\lambda)=P_{i-1}(\lambda)=1(i=0,1, \cdots, m ; j=0,1, \cdots, n)$.

LEMMA 2.

$$
\bar{\lambda}_{i j}<2, \quad i=0,1, \cdots, m ; j=0,1, \cdots, n .
$$

We shall prove this lemma by showing that

$$
P_{i j}(\lambda) \geqq P_{i-1}{ }_{j}(\lambda) \cdot P_{i j-1}(\lambda)>0, \quad \lambda \geqq 2 .
$$


We establish this result by induction. It is seen that the above is true for $i+j=0$ and 1 . Now

$$
\begin{array}{ll}
P_{i 0}(\lambda)=\lambda P_{i-10}(\lambda)-\frac{1}{2} P_{i-20}(\lambda), & \\
P_{i 0}(\lambda) \geqq\left(\lambda-\frac{1}{2}\right) P_{i-10}(\lambda)>0, & \lambda \geqq 2, \\
P_{i 0}(\lambda) \geqq P_{i-10}(\lambda)>0, & \lambda \geqq 2 .
\end{array}
$$

And since $P_{0 j}(\lambda)=P_{j 0}(\lambda)$ it follows that

Now

$$
P_{0 j}(\lambda) \geqq P_{0 j-1}(\lambda)>0, \quad \lambda \geqq 2 .
$$

$$
\begin{array}{rlrl}
P_{i j}(\lambda)= & \lambda P_{i-1 j}(\lambda) P_{i j-1}(\lambda)-\frac{1}{2} P_{i j-1}(\lambda) P_{i-1 j-1}(\lambda) P_{i-2}(\lambda) & & \\
& -\frac{1}{2} P_{i-1 j}(\lambda) P_{i-1} i_{-1}(\lambda) P_{i j-2}(\lambda), & & \\
P_{i j}(\lambda) \geqq & (\lambda-1) P_{i-1} j_{j}(\lambda) P_{i j-1}(\lambda)>0, & & \lambda \geqq 2, \\
P_{i j}(\lambda) \geqq P_{i-1 j}(\lambda) P_{i j-1}(\lambda)>0, & & \lambda \geqq 2 .
\end{array}
$$

Hence $\bar{\lambda}_{i j}<2(i=0,1, \cdots, m ; j=0,1, \cdots, n)$. From Theorem 1 and Lemma 2 we have immediately the following result.

THEOREM 2. If $c_{00}>0$,

$$
\begin{aligned}
& c_{2 i 2 j} c_{2 i+22 j}-4 c_{2 i+12 j}^{2}>0 \text {, } \\
& i=0,1, \cdots, m-1 ; j=0,1, \cdots, n-1, \\
& c_{2 i 2 n} c_{2 i+2}{ }_{2 n}-2 c_{2 i+12 n}^{2}>0, \quad i=0,1, \cdots, m-1 \text {, } \\
& c_{2 i 2 j} c_{2 i 2 j+2}-4 c_{2 i 2 j+1}^{2}>0, \\
& i=0,1, \cdots, m-1 ; j=0,1, \cdots, n-1, \\
& c_{2 m 2 j} c_{2 m 2 j+2}-2 c_{2 m 2 j+1}^{2}>0, \quad j=0,1, \cdots, n-1 \text {, } \\
& c_{2 i+12 j+1}=0, \quad i=0,1, \cdots, m-1 ; j=0,1, \cdots, n-1,
\end{aligned}
$$

then $L(x, y)>0$ for all real values of $x$ and $y$.

This theorem is a generalization of the theorem of E. B. Van Vleck ${ }^{2}$ for the case of a polynomial in a single real variable $x$.

4. Polynomials in several real variables. Let

(1) $\quad T\left(x_{1}, x_{2}, \cdots, x_{n}\right)=\sum_{p=0}^{2 u} \sum_{q=0}^{2 v} \cdots \sum_{r=0}^{2 w} b_{p q} \ldots r c_{p q} \ldots r x_{1}^{p} x_{2}^{q} \ldots x_{n}^{r}$,

${ }^{2}$ E. B. Van Vleck, $A$ sufficient condition for the maximum number of imaginary roots of an equation of the nth degree, Annals of Mathematics (2), vol. 4 (1902-1903), p. 191. 
where the $b_{p q} \ldots r$ are positive constants, and the $c_{p q} \ldots r$ are real numbers. Let $A$ denote the class of all the distinct permutations $i j \cdots k$ where $i$ ranges from 0 to $u, j$ ranges from 0 to $v, \cdots$, and $k$ ranges from 0 to $w$. We define the following $n+1$ functions over the class $A$

$$
\begin{aligned}
I_{i j \cdots k}=\begin{array}{l}
1, i \neq u \\
0, i=u
\end{array} J_{i j \cdots k}=\begin{array}{l}
1, j \neq v \\
0, j=v
\end{array}, \cdots, K_{i j \cdots k}=\begin{array}{l}
1, k \neq w \\
0, k=w
\end{array} \\
S_{i j \cdots k}=\frac{n}{n-d_{i j \cdots k}}, \quad i j \cdots k \neq u v \cdots w, \\
S_{u v \cdots w}=n,
\end{aligned}
$$

where $d_{i j \ldots k}$ represents the number of the indices $i, j, \ldots, k$ which are equal to $u, v, \cdots, w$, respectively. Introducing parameters we rewrite the polynomial (1) as

$$
\begin{aligned}
& T\left(x_{1}, x_{2}, \cdots, x_{n}\right)=\sum_{A}\left[S_{i j} \ldots{ }_{k} \alpha_{i j} \ldots_{k} c_{2 i 2 j} \ldots 2 k x_{1}^{2 i} x_{2}^{2 j} \cdots x_{n}^{2 k}\right. \\
& \left\{I_{i j \ldots k}\left(1+\beta_{i j \ldots k} \frac{c_{2 i+12 j \cdots 2 k}}{S_{i j \ldots k} c_{2 i 2 j \cdots 2 k}} x_{1}\right)^{2}\right. \\
& +J_{i j \cdots k}\left(1+\gamma_{i j \ldots k} \frac{c_{2 i} 2 j+1 \cdots 2 k}{S_{i j \ldots k} c_{2 i 2 j \cdots 2 k}} x_{2}\right)^{2}+\cdots \\
& \left.+K_{i j \ldots k}\left(1+\delta_{i j \ldots k} \frac{c_{2 i 2 j} \ldots 2 k+1}{S_{i j \ldots k} c_{2 i 2 j} \ldots 2 k} x_{n}\right)^{2}\right\}+I_{i j \ldots k} \zeta_{i j \cdots k} \\
& \frac{\left(1 / \lambda^{2}\right) c_{2 i 2 j \cdots 2 k} c_{2 i+22 j \cdots 2 k}-c_{2 i+12 j \cdots 2 k}^{2} / S_{i j \cdots k}}{c_{2 i 2 j} \cdots 2 k} x_{1}^{2 i+2} x_{2}^{2 j} \cdots x_{n}^{2 k}
\end{aligned}
$$

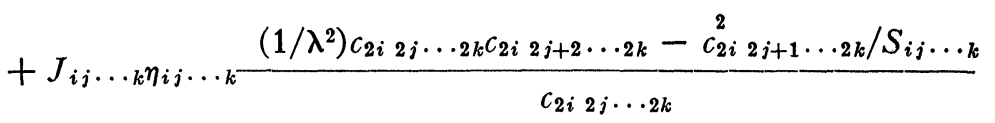

$$
\begin{aligned}
& \cdots x_{1}^{2 i} \cdots x_{2}^{2 j+2} \cdot x_{n}^{2 k}+\cdots+K_{i j} \cdots k \mu_{i j} \cdots k \\
& \left.\frac{\left(1 / \lambda^{2}\right) c_{2 i} c_{2 j \ldots 2 k} c_{2 i}{ }_{2 j} \ldots 2 k+2-c_{2 i 2 j}^{2} \ldots 2 k+1}{c_{2 k}} S_{i j \ldots k} x_{1}^{2 i} x_{2}^{2 j} \cdots x_{n}^{2 k+2}\right] \\
& +\sum_{p=0}^{u-1} \sum_{q=0}^{v-1} \cdots \sum_{r=0}^{w-1} b_{2 p+1} 2 q+1 \cdots 2 r+1 c_{2 p+1} 2 q+1 \cdots 2 r+1 x_{1}^{2 p+1} x_{2}^{2 q+1} \cdots x_{n}^{2 r+1} \text {, }
\end{aligned}
$$


where $\sum_{A}$ means that the summation shall extend over all permutations $i j \cdots k \in A$. By comparing coefficients we obtain

$$
\begin{aligned}
& n \alpha_{00} \cdots_{0}=b_{00} \ldots 0 \text {, } \\
& 2 \alpha_{i j \ldots k} \beta_{i j} \ldots k=b_{2 i+12 j} \ldots 2 k \text {, } \\
& 2 \alpha_{i j} \ldots k \gamma_{i j} \ldots k=b_{2 i 2 j+1} \ldots 2 k \text {, } \\
& 2 \alpha_{i j \ldots k} \delta_{i j \ldots k}=b_{2 i 2 j \ldots 2 k+1} \text {, } \\
& \zeta_{i j \ldots k}=\alpha_{i j \ldots k} \beta_{i j \ldots k}^{2} \text {, } \\
& \eta_{i j} \ldots k=\alpha_{i j} \ldots k \gamma_{i j}^{2} \ldots k \text {, } \\
& \mu_{i j \ldots k}=\alpha_{i j \ldots k} \delta_{i j \ldots k}^{2}, \\
& n \alpha_{i j \ldots k}+\frac{1}{\lambda^{2}}\left(\zeta_{i-1} j_{\ldots k}+\eta_{i j-1} \ldots k+\cdots+\mu_{i j \ldots k-1}\right)=b_{2 i 2 j \cdots 2 k} \text {, }
\end{aligned}
$$

where we define $\zeta_{i j \ldots k}=\eta_{i j \ldots k}=\cdots=\mu_{i j \ldots k}=0, \alpha_{i j} \ldots k=1, i j \cdots k$ $\notin A ; b_{p q} \cdots r=0$ if $p, q, \cdots, r$ are not in the ranges 0 to $2 u, 0$ to $2 v, \cdots, 0$ to $2 w$, respectively. The parameters $\alpha_{i j} \ldots k$ are determined by the recursion relations

$$
\begin{aligned}
& n \alpha_{00} \ldots 0=b_{00} \ldots 0,
\end{aligned}
$$

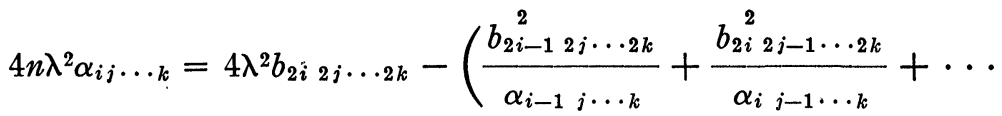

$$
\begin{aligned}
& \left.+\frac{b_{2 i 2 j \cdots 2 k-1}^{2}}{\alpha_{i j} \ldots k-1}\right) \text {. }
\end{aligned}
$$

We write $\alpha_{i j \ldots k}=P_{i j \ldots k}(\lambda) / n \lambda Q_{i j \ldots k}(\lambda)$, where $P_{i j \ldots k}(\lambda)$ and $Q_{i j \ldots k}(\lambda)$ are polynomials in $\lambda$. The polynomials $P_{i j \ldots k}(\lambda)$ and $Q_{i j} \ldots k(\lambda)$ satisfy the recursion relations

$$
\begin{aligned}
& P_{00} \ldots 0(\lambda)=b_{00} \ldots 0 \lambda, \quad Q_{00} \ldots 0(\lambda)=1, \\
& P_{i j \ldots k}(\lambda)=Q_{i j \ldots k}(\lambda)\left\{\lambda b_{2 i 2 j \ldots 2 k}-\frac{n}{4}\left[\frac{b_{2 i-12 j \cdots 2 k}^{2} Q_{i-1 j} \ldots k}{(\lambda)}\right.\right.
\end{aligned}
$$

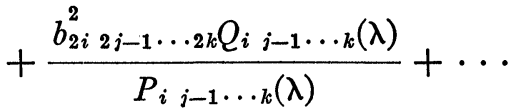

$$
\begin{aligned}
& \left.\left.+\frac{b_{2 i 2 j \cdots 2 k-1}^{2} Q_{i j \cdots k-1}(\lambda)}{P_{i j \cdots k-1}(\lambda)}\right]\right\} \text {, } \\
& Q_{i j \ldots k}(\lambda)=P_{i-1} j_{j k}(\lambda) P_{i j-1} \ldots k(\lambda) \cdots P_{i j \ldots k-1}(\lambda) \text {, }
\end{aligned}
$$


where we define $P_{i j \ldots k}(\lambda)=Q_{i j \ldots k}(\lambda)=1$ for $i j \cdots k \notin A$. Let $\bar{\lambda}_{i j \ldots k}$ be the greatest positive zero of $P_{i j} \ldots k(\lambda)$. Then we can establish the following lemma.

LEMmA 3. $\bar{\lambda}_{i j \ldots k}$ is greater than or equal to the greater of $\bar{\lambda}_{i-1} j_{\ldots k}$, $\bar{\lambda}_{i j-1} \ldots k, \cdots, \bar{\lambda}_{i j} \ldots k-1$.

The proof of this lemma is similar to that given for Lemma 1.

THEOREM 3. Let $r$ be a real constant. If $r \geqq \bar{\lambda}_{u v} \ldots w$, and

$$
\begin{aligned}
& c_{00 \cdots 0}>0, \\
& \frac{1}{r^{2}} c_{2 i 2 j \cdots 2 k} c_{2 i+2} 2 j \cdots 2 k-\frac{c_{2 i+12 j \cdots 2 k}^{2}}{c_{i j \cdots k}}>0, \\
& \frac{1}{r^{2}} c_{2 i 2 j \cdots 2 k} c_{2 i 2 j+2 \cdots 2 k}-\frac{c_{2 i 2 j+1 \cdots 2 k}^{2}}{S_{i j \cdots k}}>0, \\
& \frac{1}{r^{2}} c_{2 i 2 j \cdots 2 k} c_{2 i 2 j \cdots 2 k+2}-\frac{c_{2 i 2 j \cdots 2 k+1}^{2}}{S_{i j \cdots k}}>0, \\
& c_{2 i+12 j+1 \cdots 2 k+1}=0,
\end{aligned}
$$

then

$$
T\left(x_{1}, x_{2}, \cdots, x_{n}\right)>0
$$

for all real values of $x_{1}, x_{2}, \cdots, x_{n}$.

This theorem follows from the preceding lemma in the same manner that Theorem 1 follows from Lemma 1.

If we take the coefficients

$$
b_{p q} \ldots r=1,
$$

we can deduce a result generalizing Theorem 2 . We consider the polynomial

$$
L\left(x_{1}, x_{2}, \cdots, x_{n}\right)=\sum_{p=0}^{2 u} \sum_{q=0}^{2 v} \cdots \sum_{r=0}^{2 w} c_{p q} \ldots r x_{1}^{p} x_{2}^{q} \cdots x_{n}^{r} .
$$

We define over the class $A$ the following $n$ functions

$$
f_{i j \ldots k}=\begin{aligned}
& 0, i=0 \\
& 1, i \neq 0
\end{aligned}, g_{i j \ldots k}=\begin{aligned}
& 0, j=0 \\
& 1, j \neq 0
\end{aligned}, \cdots, h_{i j \ldots k}=\begin{aligned}
& 0, k=0 \\
& 1, k \neq 0
\end{aligned} .
$$

The recursion relations that the polynomials $P_{i j \ldots k}(\lambda)$ satisfy in this case are 


$$
\begin{aligned}
& P_{00 \ldots 0}(\lambda)=\lambda \\
& P_{i j \ldots k}(\lambda)=Q_{i j \ldots k}(\lambda)\left(\lambda-\frac{n}{4}\left[f_{i j \ldots k} \frac{Q_{i-1 j \ldots k}(\lambda)}{P_{i-1 j \ldots k}(\lambda)}\right.\right.
\end{aligned}
$$

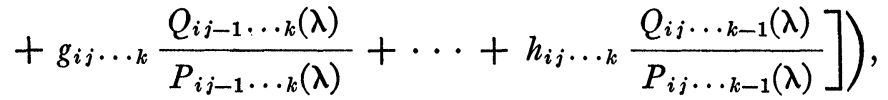

$$
\begin{aligned}
& Q_{i j \ldots k}(\lambda)=P_{i-1 j \ldots k}(\lambda) P_{i j-1} \ldots k(\lambda) \cdots P_{i j \ldots k-1}(\lambda) \text {, }
\end{aligned}
$$

where we have defined $P_{i j \ldots k}(\lambda)=Q_{i j \ldots k}(\lambda)=1$ for $i j \cdots k \notin A$.

LEMma $4 . \bar{\lambda}_{i j} \ldots k<n^{2} / 4+1$.

The proof of this lemma is similar to that given for Lemma 2.

THEOREM 4 . If $c_{00} \ldots 0>0$, and

$$
\begin{aligned}
& \frac{16}{\left(n^{2}+4\right)^{2}} c_{2 i 2 j \cdots 2 k} c_{2 i+22 j \cdots 2 k}-\frac{\stackrel{2}{c_{2 i+12 j} \ldots 2 k}}{S_{i j} \ldots k}>0, \\
& \frac{16}{\left(n^{2}+4\right)^{2}} c_{2 i 2 j \cdots 2 k} c_{2 i 2 j+2 \cdots 2 k}-\frac{c_{2 i 2 j+1}^{2} \ldots 2 k}{S_{i j \ldots k}}>0 \text {, }
\end{aligned}
$$

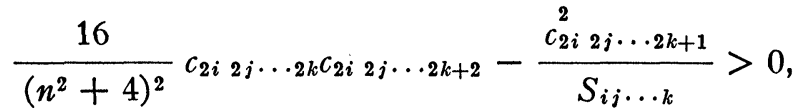

$$
\begin{aligned}
& c_{2 i+12 j+1} \cdots 2 k+1=0,
\end{aligned}
$$

then $L\left(x_{1}, x_{2}, \cdots, x_{n}\right)>0$ for all real values of $x_{1}, x_{2}, \cdots, x_{n}$.

ILlinois Institute of TEChNOLOGY 